\title{
Mochilas escolares y dolor de espalda en la población infantil
}

\author{
S. Alberola López ${ }^{\mathrm{a}}$, I. Pérez García ${ }^{\mathrm{a}}$, I. Casares Alonsob, \\ A. Cano Garcinuño ${ }^{c}$, JM. Andrés de Llano ${ }^{d}$ \\ aPediatra. CS Jardinillos. Palencia. España. \\ bPediatra. CS de Venta de Baños. Palencia. España. \\ cPediatra. CS de Villamuriel de Cerrato. Palencia. España. \\ dPediatra. Complejo Asistencial. Palencia. España.
}

\section{Resumen}

Introducción: el peso de las mochilas preocupa por el esfuerzo físico que realizan los niños y su relación con problemas de espalda. El objetivo es conocer los hábitos del uso de mochilas y su relación con el dolor de espalda en población escolar.

Material y métodos: estudio observacional en 4 consultas pediátricas. Se analizan variables demográficas, antropométricas y se administra un cuestionario.

Resultados: son 159 niños, 80 varones y 79 mujeres, de 11 y 14 años, que cursan $5^{\circ}$ de

Educación Primaria (EP) y $2^{\circ}$ de Educación Secundaria (ESO). El $69 \%$ acude al colegio andando, el $80 \%$ lleva la mochila en ambos hombros, el 59\% se cansa y el 62,3\% refiere dolor de espalda. La puntuación del dolor es 5 (escala 0-10).

La mochila pesa $6,3 \pm 2 \mathrm{~kg}$. El peso relativo (porcentaje de peso en relación al peso del niño) es $13,4 \% \pm 5,5$ y no difiere entre sexos, medio urbano o rural, pero sí entre grupos de edad (15,5\% en EP, $11,6 \%$ en ESO, $p<0,001$ ) y centro (público: $14,3 \%$; concertado: $12,3 \% ; p=$ $0,02)$. No encontramos diferencias en el peso con o sin dolor $(13,8 \%$ versus $12,7 \% ; p=0,19)$ ni en la presencia de dolor por sexo o edad. No se encuentra asociación entre dolor y horas de tele/ videojuegos pero sí entre dolor y horas de deporte extraescolar (más horas, menos dolor: OR [odds ratio]: 0,23; IC [intervalo de confianza] 95\%: 0,08-0,7). Existe diferencia en la puntuación de síntomas psicosomáticos, mayor en los que sufren dolor (OR 1,37; IC 95\%: 1,2-1,6).

Conclusiones: el peso de las mochilas es el 13,4\% $\pm 5,5 \mathrm{del}$ peso del niño. Aunque no encontramos asociación entre dolor y peso, este influye en la sensación de malestar en la infancia y debe considerarse un problema de salud.

Palabras clave: Mochila escolar. Dolor de espalda. Infancia.

School backpacks and back pain in child population

Abstract

Introduction: backpacks' load is worrisome because of the physical effort made by children and its potential relationship with back problems.

Susana Alberola López: salberola@ono.com

Los autores declaran no presentar conflictos de intereses en relación con la preparación y publicación de este artículo.

Algunos datos de este estudio fueron presentados en forma de comunicación oral en la 5. ${ }^{a}$ Reunión Anual de la AEPap y 5. ${ }^{a}$ Reunión de la ArAPAP en Zaragoza los pasados 13 y 14 de noviembre de 2009. 
Objectives: to know the habits of school bags' use and its relationship with back pain in the paediatric school population.

Material and methods: cross-sectional observational study in three primary care centres. We analyze anthropometric and demographic variables and we fill in a questionnaire based on previous studies.

Results: one hundred and fifty-nine children were included. They were 80 males y 79

females, aged 11 and 14 years, studying $5^{\circ}$ year in primary level (EPO) and $2^{\circ}$ year in secondary level (ESO). Sixty-nine percent of them walk to school, $80 \%$ carry the backpack in both shoulders, 59\% feel tired with it, and $62.3 \%$ refer back pain. The mean score of pain is 5 (scale: 0 -10). Schoolbag weight is $6.3 \mathrm{~kg} \pm 2$. Relative weight is $13.4 \% \pm 5.5$ of the children's body weight and it is not different neither between genders, nor in urban-rural site, but differs between ages $\left(15.5 \%\right.$ in $5^{\circ}$ EPO, $11.6 \%$ in $2^{\circ}$ ESO; $\left.p<0.001\right)$ and type of school (public: 14.3\%; concerted: $12.3 \% ; p=0.02$ ). The relative weight is higher in those students who feel that the backpack is too heavy $(14.2 \%$ versus $12.2 \% ; p=0.02)$, but it is not in those who refer back pain (13.8\% versus $12.7 \% ; p=0.19)$. We have not found neither differences in back pain with gender and age, nor association between pain and number of TV/videogames watching hours. There is association between pain and number of extra-school sports hours (the more hours, the less pain: OR [odds ratio]: 0.23; Cl [confidence interval] 95\%:0.08-0.7). There is a difference in the psychosomatic symptoms score (scale: 0-18), being higher in those who have back pain (OR 1.37; CI 95\%: 1.2-1.6).

Conclusions: the schoolbags' relative weight is $13.4 \% \pm 5.5$. Although we have not found association between back pain and backpack weight, it affects the feeling of discomfort in childhood and it must be considered a health problem.

Key words: Schoolbag. Back pain. Childhood.

\section{Introducción}

El uso de mochilas escolares es una actividad que forma parte de la vida diaria de la población infantil española. En ellas se transportan los objetos necesarios para el desarrollo de las tareas en las aulas (libros, cuadernos, carpetas, estuches), alimentos y bebidas para consumir en el recreo, útiles de aseo, así como utensilios variados $y$ tal vez prescindibles durante el tiempo de permanencia en la escuela (videojuegos, otros juguetes, teléfonos móviles, tebeos, libros de lectura, etc.). Con todo, son los elementos relacionados con la actividad escolar los responsables máximos del peso de la mochila, que a menudo se considera excesivo por alumnos, padres y profesores.

La preocupación actual por el peso de las mochilas se debe no solo al esfuerzo físico que necesitan realizar los niños para transportarlas, sino también a su posible relación con el dolor de espalda y con las alteraciones estáticas de la columna vertebral en el momento presente $y$ en la edad adulta ${ }^{1-7}$. Existen diversos estudios que tratan de estable- 
cer una cantidad de peso segura para que niños y adolescentes la lleven de forma habitual y que, según los autores, oscila entre el 10 y el $20 \%$ del peso corporal $^{8-13}$. En 1996, el Ministerio de Educación de Austria decidió no permitir que las mochilas escolares pesasen más de un $10 \%$ del peso del niño ${ }^{1,4}$. En Alemania existe una reglamentación similar. Desconocemos si existe alguna legislación parecida en otros países de nuestro entorno.

Esa inquietud se ve reflejada también en el creciente número de trabajos publicados en los últimos años sobre la relación entre el uso de mochilas y las lesiones agudas, el dolor de espalda (también de cuello y de hombro), la postura y la curvatura espinal, la función pulmonar, etc. ${ }^{1-10,14-16}$. Sin embargo, hay pocas referencias de publicaciones que hayan sido realizadas sobre este tema en España ${ }^{17-19}$. En nuestro estudio nos centraremos en el dolor de espalda como síntoma a considerar.

En el pasado, el dolor de espalda en la infancia era considerado tan poco frecuente que su existencia obligaba a realizar estudios más detallados para descartar una causa orgánica como responsable del mismo. Sin embargo, en la bibliografía reciente se pone de manifies- to que la prevalencia del dolor oscila, según las series, entre el 8 y el $74 \%$, con diferencias según las edades estudiadas y la definición de dolor ${ }^{1-6}$. A pesar de este rango tan amplio, se ha evidenciado un aumento en todas las edades, en especial en torno a la pubertad, en relación con diversos factores epidemiológicos, biomecánicos y fisiológicos, entre los que destacamos el pico máximo de crecimiento que se produce en este tramo de edad"11.

El objetivo de nuestro trabajo es conocer las características del uso de las mochilas escolares y su relación con el dolor de espalda en una población infantil de nuestro entorno. Asimismo, queremos conocer la relación entre el dolor de espalda, el nivel de actividad física y las características psicosomáticas de esta población.

\section{Material y métodos}

Se trata de un estudio observacional de tipo transversal, sobre la población escolar de 11 y 14 años atendida en tres centros de salud de la provincia de Palencia, uno urbano (Jardinillos) y dos semiurbanos (Venta de Baños y Villamuriel de Cerrato), que acuden a las actividades preventivas y de promoción de la salud programadas desde octubre de 2007 a abril de 2009. 
Los criterios de exclusión son: negarse a participar en el estudio, no responder a las preguntas del cuestionario elaborado para tal fin y pacientes que por problemas físicos no porten habitualmente la mochila.

Las variables de medida son: datos demográficos (nombre, fecha de naci-

Tabla 1. Cuestionario sobre el uso de mochilas escolares

Nombre / Sexo: M_F / Fecha de nacimiento / Edad.

Curso escolar / Jornada: C_P / Centro Escolar.

Peso / Talla / IMC / Peso de la mochila.

1. ¿Cómo vas al colegio? Coche_Autobús_Moto_Bicicleta_Andando.

2. ¿Cuánto tiempo (en minutos) tardas en llegar de casa al colegio? $<5,5-10,10-15,15-20,20-30,>30$.

3. ¿Cuánto tiempo (en minutos) llevas tú personalmente tu mochila? $<5,5-10,10-15,15-20,20-30,>30$.

4. ¿Cómo llevas normalmente la mochila? En ambos hombros_En un hombro_Con la mano_Ruedas.

5. ¿Te sientes cansado llevando tu mochila? Nunca_Casi nunca_Algunas veces_A menudo_Siempre.

6. ¿Piensas que tu mochila es demasiado pesada para ti? Sí_No.

7. ¿Alguna vez te duele la espalda? Sí_No (si la respuesta es NO, pasar a la 14).

8. ¿Con qué frecuencia te duele?

Una o dos veces_Al menos, una al mes_Al menos, una a la semana_Una al día.

9. ¿Desde cuándo te duele? Menos de un mes_1-3 meses_3-6 meses_6 o más.

10. ¿Cómo de intenso es el dolor? (puntúa de 1 a 10).

11. ¿Has perdido colegio, otras clases o deportes por el dolor? Sí_No.

12. ¿Has ido al médico por este dolor? Sí_No.

13. Si te duele, ¿el dolor empeora si haces algo de lo siguiente? Llevando la mochila_Levantando la mochila_Corriendo.

14. ¿Cuántas horas ves la tele o juegas con videojuegos al día (de promedio)? 0_1_2_3_4_+5

15. ¿Cuántas horas dedicas a actividades deportivas extraescolares semanalmente? 0_1_2_3_4_+5

16. ¿Alguna vez te sientes apático? (sin apetecerte hacer nada en absoluto) Nunca_Algunas veces_Con frecuencia.

17. ¿Alguna vez te sientes cansado sin saber exactamente por qué? Nunca_Algunas veces_Con frecuencia.

18. ¿Alguna vez te duele la cabeza porque estás estresado? Nunca_Algunas veces_Con frecuencia.

19. ¿Alguna vez no tienes apetito? Nunca_Algunas veces_Con frecuencia.

20. ¿Tienes dificultades para dormirte? Nunca_Algunas veces_Con frecuencia.

21. ¿Tienes la piel sensible y fácilmente te salen erupciones, manchas o te pica? Nunca_Algunas veces_Con frecuencia.

22. ¿Alguna vez te duele el estómago alrededor del ombligo? Nunca_Algunas veces_Con frecuencia.

23. ¿Alguna vez notas que no puedes relajar tus músculos adecuadamente? Nunca_Algunas veces_Con frecuencia.

24. ¿Alguna vez lloras mucho sin que haya un motivo real para hacerlo? Nunca_Algunas veces_Con frecuencia. 
miento, sexo), datos escolares (centro, curso, tipo de jornada continua/partida, peso de la mochila), datos físicos (peso, talla, índice de masa corporal).

El pediatra entrega un cuestionario de 24 preguntas para responder en su domicilio (tabla 1), basado en los estudios de Negrini y Carabalona ${ }^{3}$, Siambanes y cols. ${ }^{2}$, van Gent y cols. ${ }^{4}$ y el test de actividad física Krece Plus ${ }^{20}$.

En las variables continuas, se utilizan como estimadores la media y la desviación estándar. Se comprueba la bondad de ajuste a la normalidad mediante el test de Kolmogorov-Smirnov. Para las comparaciones entre grupos, se utiliza el test de la t de Student o el análisis de la varianza en las variables que siguen una distribución normal. Cuando no es así, se utilizan los test de Mann-Whitney y Kruskal-Wallis.

En las variables discretas y categóricas, se obtiene la distribución de frecuencias con porcentajes. Para las comparaciones entre grupos, se utilizan tablas de contingencia con el test de chicuadrado y la prueba exacta de Fisher cuando es preciso.

Finalmente, para determinar el grado de asociación entre las distintas varia-

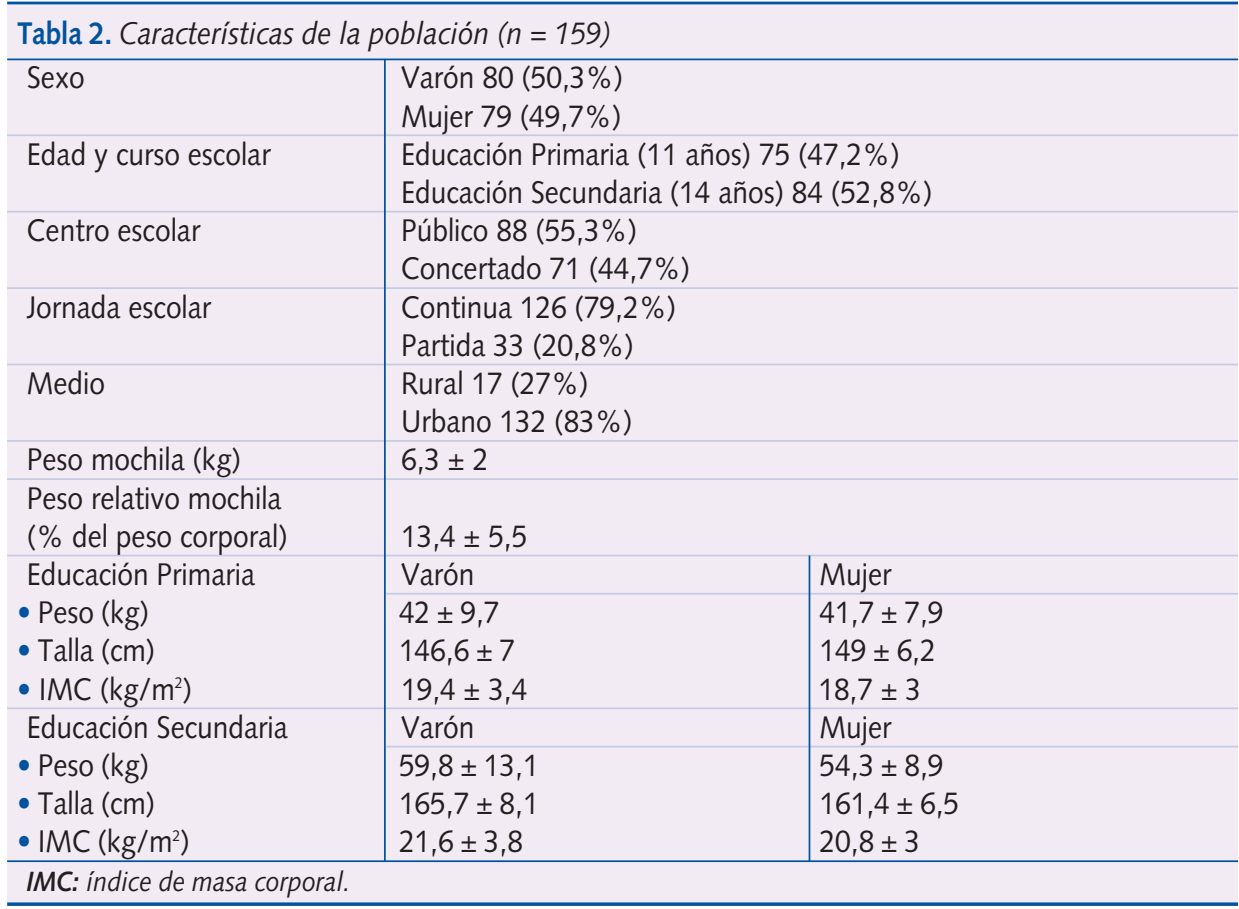


bles y controlar las potencialmente confusoras, se realiza análisis multivariante, a través de la regresión lineal múltiple y regresión logística binaria.

\section{Resultados}

De los 322 niños y adolescentes que realizaron las revisiones de salud de $11 \mathrm{y}$ 14 años en el periodo considerado, 159 contestaron el cuestionario (49,4\%). Las características de la población estudiada se recogen en la tabla 2.

La tabla 3 muestra los hábitos de desplazamiento al colegio y uso de la mochila (preguntas 1 a 6). En la tabla 4 se hace referencia a las características del dolor de espalda (preguntas 7 a 13).
Respecto a otros hábitos de esta población escolar, casi el $90 \%$ ve la televisión o juega con videojuegos entre 1 y 3 horas cada día y el $83,6 \%$ practica entre 1 y 5 horas semanales de deporte extraescolar.

Se ha formulado también un bloque de 8 preguntas (16 a 24) destinadas a explorar factores psicosomáticos, que se puntúan: 0 (nunca), 1 (algunas veces) y 2 (siempre). Sobre una puntuación máxima posible de 18, la mediana de nuestro grupo es 4.

Considerando el peso de la mochila, expresado como el porcentaje que supone sobre el peso corporal del niño, no hemos encontrado diferencias significa-

\begin{tabular}{|c|c|c|}
\hline \multirow{4}{*}{ ¿Cómo vas al colegio? } & Coche & $21(13,2 \%)$ \\
\hline & Autobús & $13(8,2 \%)$ \\
\hline & Andando & $109(68,6 \%)$ \\
\hline & Vehículo/Andando & $16(10,1 \%)$ \\
\hline \multirow[t]{5}{*}{ ¿Cuánto tardas? } & $<5$ minutos & $32(20,1 \%)$ \\
\hline & 5-10 minutos & $63(39,6 \%)$ \\
\hline & $10-15$ minutos & $35(22 \%)$ \\
\hline & $15-20$ minutos & $17(10,7 \%)$ \\
\hline & $>20$ minutos & $12(7,5 \%)$ \\
\hline \multirow[t]{3}{*}{ ¿Cómo llevas la mochila? } & En ambos hombros & $127(79,9 \%)$ \\
\hline & En un hombro & $8(5 \%)$ \\
\hline & Con ruedas & $23(14,5 \%)$ \\
\hline \multirow[t]{5}{*}{ ¿Te sientes cansado llevando la mochila? } & Nunca & $31(19,5 \%)$ \\
\hline & Casi nunca & $34(21,4 \%)$ \\
\hline & Algunas veces & $68(42,8 \%)$ \\
\hline & A menudo & $16(10,1 \%)$ \\
\hline & Siempre & $9(5,7 \%)$ \\
\hline \multirow[t]{2}{*}{ ¿Piensas que es demasiado pesada? } & No & $67(42,1 \%)$ \\
\hline & Sí & $92(57,9 \%)$ \\
\hline
\end{tabular}


tivas entre sexos, tipo de jornada escolar y medio rural o urbano. Sí existen entre cursos escolares (Educación Primaria [EP]: 15,5\%; Educación Secundaria $[E S]: 11,5 \% ; p<0,001)$ y tipos de centro (público: 14,3\%; concertado: $12,3 \% ; p=0,02)$.

En la pregunta 14 sobre horas diarias de televisión y videojuegos, hemos encontrado diferencias significativas en la edad de los escolares $(p=0,028)$, con más horas en los mayores. También se encuentran en la pregunta 15 sobre las horas semanales de deporte extraesco$\operatorname{lar}(p<0,001)$, a favor de los varones.
En el bloque de preguntas sobre factores psicosomáticos (16 a 24), no hemos hallado correlación con edad, sexo, medio rural o urbano, centro público o concertado, jornada continua o partida, ni con el peso de la mochila.

La tabla 5 se centra en el dolor de espalda. No encontramos diferencias entre presencia de dolor y edad, sexo, medio urbano o rural, tipo de centro y peso de la mochila.

Tampoco existen en el número de horas diarias de televisión y videojuegos, pero sí en las horas semanales de deporte extraescolar $(p=0,004)$, más nu-

\begin{tabular}{|c|c|c|}
\hline \multirow[t]{2}{*}{ ¿Alguna vez te duele la espalda? } & No & $60(37,7 \%)$ \\
\hline & Sí & $99(62,3 \%)$ \\
\hline \multirow[t]{4}{*}{ ¿Con qué frecuencia te duele? } & Una o dos veces & $20(12,6 \%)$ \\
\hline & Al menos, $1 \mathrm{vez} / \mathrm{mes}$ & $31(19,5 \%)$ \\
\hline & Al menos, 1 vez/semana & $36(22,6 \%)$ \\
\hline & Una vez al día & $12(7,5 \%)$ \\
\hline \multirow[t]{4}{*}{ ¿Desde cuándo te duele? } & Menos de un mes & $13(8,2 \%)$ \\
\hline & 1-3 meses & $19(11,9 \%)$ \\
\hline & 3-6 meses & $12(7,5 \%)$ \\
\hline & Más de 6 meses & $51(32,1 \%)$ \\
\hline \multirow[t]{7}{*}{ Intensidad del dolor } & 2 & $10(10,3 \%)$ \\
\hline & 3 & $12(12,4 \%)$ \\
\hline & 4 & $19(19,6 \%)$ \\
\hline & 5 & $21(21,6 \%)$ \\
\hline & 6 & $17(17,5 \%)$ \\
\hline & 7 & $9(9,2 \%)$ \\
\hline & 8 & $8(6,2 \%)$ \\
\hline \multirow[t]{2}{*}{ ¿Has perdido clase por el dolor? } & No & $96(97 \%)$ \\
\hline & Sí & $3(3 \%)$ \\
\hline \multirow[t]{2}{*}{ ¿Has ido al médico por el dolor? } & No & $82(82,8 \%)$ \\
\hline & Sí & $17(17,2 \%)$ \\
\hline
\end{tabular}


merosas en los que no tienen dolor $(3,5$ y 2,6 , respectivamente).

También existen diferencias en la puntuación de los factores psicosomáticos: mayor en los niños con dolor de espalda (5 frente a 2,8; $p<0,001$ ). Asimismo, encontramos asociación ( $p=$ 0,005) entre la puntuación psicosomática y la intensidad del dolor, pero no así entre la intensidad del dolor y el peso de la mochila.

Se realizaron modelos de regresión logística binaria para la variable dependiente "dolor de espalda". El modelo final obtenido incluye únicamente las variables: puntuación psicosomática y horas de deporte extraescolar $(p<0,001)$. La estimación de riesgo obtenida mediante odds ratio (OR) fue de 1,37 (intervalo de confianza [IC] del 95\%: 1,2$1,6)$ para cada punto obtenido en la variable "puntuación psicosomática", ajustado por la variable de deporte extraescolar. La OR obtenida para la variable "número de horas semanales de deporte extraescolar" fue de 0,23 (IC 95\%: 0,08-0,7) para los que realizaban 4 y 5 horas de deporte extraescolar, ajustado por la variable de puntuación psicosomática.

\section{Discusión}

El uso de mochilas escolares es una actividad habitual en los niños y adolescentes, y supone un esfuerzo físico cotidiano nada desdeñable. En nuestro trabajo hemos querido conocer las características del uso de dichas mochilas y su relación con el dolor de espalda en una población infantil de nuestro entorno.

Los estudiantes de nuestra población tienen 11 y 14 años, tramo etario en el que se centran la mayoría de los trabajos publicados sobre este tema.

\begin{tabular}{|c|c|c|c|c|}
\hline \multicolumn{5}{|c|}{ Tabla 5. Comparación para la variable "dolor" } \\
\hline \multirow{2}{*}{\multicolumn{2}{|c|}{ Variables }} & \multicolumn{2}{|c|}{ Dolor } & \multirow[b]{2}{*}{ Significación } \\
\hline & & Sí & No & \\
\hline \multirow[t]{2}{*}{ Sexo } & Varón (\%) & $48(60 \%)$ & $32(40 \%)$ & \\
\hline & Mujer (\%) & $51(64,5 \%)$ & $28(35,5 \%)$ & $p=0,6$ \\
\hline \multicolumn{2}{|c|}{ Curso escolar: } & & & \\
\hline \multicolumn{2}{|c|}{ Primaria (11 años) } & $45(60 \%)$ & $30(40 \%)$ & \\
\hline \multicolumn{2}{|c|}{ Secundaria (14 años) } & $54(64,3 \%)$ & $30(35,7 \%)$ & $p=0,6$ \\
\hline \multicolumn{2}{|c|}{$\%$ de peso de la mochila $(M \pm D E)$} & $12,7 \pm 5$ & $13,8 \pm 5,7$ & $p=0,19$ \\
\hline \multicolumn{2}{|c|}{ Horas televisión al día $(\mathrm{M} \pm \mathrm{DE})$} & $2,2 \pm 1$ & $1,9 \pm 0,9$ & $p=0,1$ \\
\hline \multicolumn{2}{|c|}{ Horas deporte a la semana $(M \pm D E)$} & $2,6 \pm 1,9$ & $3,6 \pm 1,7$ & $p=0,003$ \\
\hline \multicolumn{2}{|c|}{ Puntuación psicosomática $(M \pm D E)$} & $5 \pm 3,1$ & $2,8 \pm 2,2$ & $p<0,001$ \\
\hline \multicolumn{5}{|c|}{ M: media. DE: desviación estándar. } \\
\hline
\end{tabular}


El peso relativo de las mochilas $(13,4 \%$ $\pm 5,5$ ) se encuentra en consonancia con los recogidos por algunos autores ${ }^{4,13,18}$, aunque discrepan de otros, por exceso $0^{1,217,19,21}$ o por defecto ${ }^{3,22}$. Estos porcentajes suponen que una parte de nuestros niños y adolescentes llevan a diario una carga superior a la recomendada por la mayoría de los estudios publicados, según razones biomecánicas y fisiológicas.

El cuestionario que han cumplimentado los escolares de nuestra población está elaborado por nosotros a partir de estudios publicados por otros autores ${ }^{2-4,20}$, de los cuales hemos seleccionado las preguntas que, a nuestro entender, mejor se ajustaban a los aspectos que queríamos investigar.

El hecho de que la mayoría de los escolares vaya al colegio andando y tarde menos de 15 minutos en llegar se debe a que el estudio se ha llevado a cabo en una ciudad pequeña (Palencia) y en 2 núcleos rurales.

Las sensaciones de cansancio y esfuerzo que la mochila produce en los niños aparecen en unos porcentajes inferiores a los encontrados por Negrini y Carabalona ${ }^{3}$, autores del cuestionario original, aunque las mochilas de su estudio eran más pesadas.

La prevalencia de dolor de espalda $(62,3 \%)$ que hemos encontrado está si- tuada en los rangos altos de los referidos en la bibliografía, que oscilan entre el 8 y el $74 \%$ 5,6. En nuestro estudio, el dolor se puede calificar de moderado (5 en una escala de 1 a 10). La medida de la intensidad del dolor no aparece en todos los estudios y suele referirse en términos relativos (Siambanes': $87 \%$ malo o muy malo; van Gent": 4,5-9,5\% grave). Nos han llamado la atención las cifras tan bajas de absentismo escolar y de consultas médicas que el dolor de espalda ocasiona. Parecen indicar que, en los niños, se asume como algo normal en su vida cotidiana, y que no merece más atención que otros problemas leves de salud. El estudio de Siambanes de donde hemos tomado estas preguntas muestra un resultado similar en lo que se refiere al escaso número de consultas que genera².

Al igual que otros autores, no hemos encontrado asociación entre la presencia de dolor de espalda y el peso de la mochila, ${ }^{4,21}$. Es un hallazgo razonable dado que la amplitud intercuartil es solamente del 5\%; por tanto, la mitad de los niños carga con un peso proporcional de mochila muy similar.

La tendencia cada vez más sedentaria de la población pediátrica se pone de manifiesto en el hecho de que casi un 10\% ve la televisión (TV) 4-5 horas to- 
dos los días y un $16,5 \%$ no hace ninguna actividad deportiva además de la educación física, incluida como asignatura obligatoria en estos niveles de educación.

Si analizamos las comparaciones entre los grupos, sólo hemos encontrado diferencias significativas en el porcentaje que supone el peso de la mochila en relación con el tipo de centro escolar (siendo mayor en los públicos, a diferencia de otros estudios en que sucede lo contrario ${ }^{23}$ ) y con el curso escolar (llevan más peso los alumnos de menor edad). Este hecho sucede en muchos casos en una época prepuberal, especialmente en los varones, con lo que su masa muscular aún no se ha desarrollado por completo y la fuerza física que pueden desplegar es todavía limitada.

Los escolares de 14 años pasan más horas delante de la TV, lo que apunta a que el sedentarismo tiende a aumentar con la edad. Los varones realizan más horas de deporte, hecho que no nos sorprende y que forma parte de nuestro entorno cultural.

En la bibliografía revisada en relación con el dolor de espalda y las mochilas escolares, aparece la asociación entre el dolor y el sexo femenino, el peso de la mochila, el medio urbano y la menor edad ${ }^{1,2,6}$, aunque también hay trabajos, como en nuestro caso, donde no se asocian dolor y peso de mochila ${ }^{4,21}$.

A diferencia de lo descrito en niños de Estados Unidos ${ }^{6}$, en los niños con dolor de espalda de nuestro estudio no hemos encontrado diferencias en el número de horas de televisión y videojuegos, al igual que en niños británicos ${ }^{21}$. Nuestros resultados con respecto a deporte extraescolar y dolor son similares a los obtenidos en niños holandeses ${ }^{4}$ en los que se encontró una asociación inversa entre horas de deporte y dolor de espalda, de tal manera que los niños que practicaban más tiempo de deporte tenían menor dolor. Por el contrario, un estudio realizado entre niños británi$\cos ^{21}$ encuentra un riesgo incrementado de dolor en aquellos con alto nivel de ejercicio físico cifrado en 6 horas semanales, aunque sin poder establecer una relación "dosis"/riesgo.

La puntuación psicosomática es mayor en los niños con dolor de espalda, resultado similar al publicado por van Gent ${ }^{4}$. $Y$ en esa misma línea, Jones encuentra asociación entre dolor de espalda, dolor somático previo y factores psicosociales adversos en población británica ${ }^{21}$.

La asociación entre la puntuación psicosomática y la intensidad del dolor, pero no entre ésta y el peso de la mochila, es un hallazgo que también refie- 
ren algunos autores ${ }^{4,21}$, aunque en la mayoría de los trabajos publicados se afirma lo contrario ${ }^{1,2,6,13}$. Este hecho nos hace pensar que el dolor de espalda no depende exclusivamente del peso soportado por el niño, sino que existen una serie de características individuales más complejas que hacen que la sensación percibida como cansancio, molestia o dolor sea diferente según los casos, y no se trate de una relación lineal y excesivamente simplista.

Según la OR obtenida, encontramos un $37 \%$ más de riesgo de dolor de espalda para cada punto que aumenta la valoración de los factores psicosomáticos, así como una protección frente al dolor 4 veces mayor si se realizan 4-5 horas semanales de deporte. En nuestro estudio, la actividad deportiva extraescolar se refiere a la que se lleva a cabo bajo la supervisión adecuada por parte de los monitores y entrenadores. Así realizada, proporciona un nivel de entrenamiento y disciplina que facilita un desarrollo armónico físico y emocional, beneficioso para la salud integral del niño. Con nuestros resultados, el deporte se comporta como un factor de protección respecto al dolor de espalda y es congruente con los planes de promoción de actividad física que programan las autoridades educativas y sanitarias.
Nuestro trabajo presenta algunas limitaciones. La primera de ellas es que se trata de un estudio transversal, de donde no se puede concluir una relación causaefecto, en el que se ha pesado la mochila una sola vez, y este peso puede no ser representativo de la realidad. Sin embargo, dado que los niños y sus familias no estaban advertidos de antemano sobre nuestra intención, asumimos que los valores no habrían sido muy diferentes de haberlo realizado en otro momento.

El estudio pregunta directamente sobre mochila y dolor, con lo que las respuestas de los niños pueden verse dirigidas en parte hacia lo que queremos estudiar, y magnificar el efecto real. Pero los estudios publicados sobre este tema adolecen del mismo defecto y no conocemos ninguna manera de enmascararlo para obviar este problema. Por otra parte, nos hubiera gustado contar con un grupo control (niños sin mochila) para realizar comparaciones entre ambos, pero no hemos podido encontrarlo.

Finalmente, los cuestionarios que hemos utilizado no están validados para su uso en español. Este hecho tiene especial importancia en el capítulo de la valoración de los síntomas psicosomáticos, donde se atribuye una puntuación determinada en función de las respuestas obtenidas. La versión original validada 
en holandé ${ }^{24}$ está fuera de nuestro alcance, pero pensamos que la traducción que hemos realizado al español, desde la versión inglesa de van Gent ${ }^{4}$, no presenta problemas de comprensión por parte de los niños. Sería deseable que se pudiera realizar en el futuro la adecuada validación para nuestro medio.

Aunque no hemos encontrado asociación entre el peso de las mochilas escolares y la presencia de dolor de espalda, creemos haber puesto de manifiesto su influencia en la sensación subjetiva de malestar de los niños y adolescentes y por ello pensamos que debe considerarse un problema de salud, porque afecta a la calidad de vida de un gran sector de nuestra población, en una etapa vulnerable.

\section{Bibliografía}

1. Skaggs DL, Early SD, D'Ambra P, Tolo VT, Kay RM. Back pain and backpacks in school children. J Pediatr Orthop. 2006;26:358-63.

2. Siambanes D, Martinez JW, Butler EW, Haider T. Influence of school backpacks on adolescent back pain. J Pediatr Orthop. 2004;24:211-7.

3. Negrini S, Carabalona R. Backpacks on! Schoolchildren's perceptions of load, associations with back pain and factors determining the load. Spine. 2002;27:187-95.

4. Van Gent C, Dols JJ, de Rover CM, Hira Sing RA, de Vet HC. The weight of schoolbags and the occurrence of neck, shoulder, and back pain in young adolescents. Spine. 2003;28: 916-21.

5. Hakala P, Rimpela A, Salminen JJ, Virtanen SM, Rimpela M. Back, neck, and shoulder pain in Finnish adolescents: national cross sectional surveys. BMJ. 2002;325:743.

6. Sheir-Neiss GI, Kruse RW, Rahman T, Jacobson LP, Pelli JA. The association of backpack use and back pain in adolescents. Spine. 2003;28:922-30.
7. Kellis $E$, Arampatzi F. Effect of sex and mode of carrying schoolbags on ground reaction forces and temporal characteristics of gait. J Pediatr Orthop B. 2009;18:275-82.

8. Chansirinukor W, Wilson D, Grimmer K, Dansie B. Effects of backpacks on students: measurement of cervical and shoulder posture. Aust J Physiother. 2001;47:110-6.

9. Grimmer K, Dansie B, Milanese S, Pirunsan $U$, Trott $P$. Adolescent standing postural response to backpack loads: a randomised controlled experimental study. BMC Musculoskelet Disord. 2002;3:10.

10. Lai JP, Jones AY. The effect of shouldergirdle loading by a school bag on lung volumes in Chinese primary school children. Early Hum Dev. 2001;62:79-86.

11. Brackley HM, Stevenson JM. Are children's backpack weight limits enough? A critical review of the relevant literature. Spine. 2004;29: 2184-90.

12. Bauer DH, Freivalds A. Backpack load limit recommendation for middle school students based on physiological and psycophysical measurements. Work. 2009;32:339-50. 
13. Viry P, Creveuil C, Marcelli C. Nonspecific back pain in children. A search for associated factors in 14-year-old schoolchildren. Rev Rhum Engl Ed. 1999;66:381-8.

14. Wiersema BM, Wall EJ, Foad SL. Acute backpack injuries in children. Pediatrics. 2003; 111:163-6.

15. Grimmer KA, Williams MT, Gill TK. The associations between adolescent head-on-neck posture, backpack weight, and anthropometric features. Spine. 1999;24:2262-7.

16. Orloff HA, Rapp CM. The effects of load carriage on spinal curvature and posture. Spine. 2004;29:1325-9.

17. Saborit N, Simó Pitarch A. Carritos o mochilas en la edad escolar. Fisioterapia. 2002;24: 63-72.

18. Ramos Espada D, González Montesinos $J$, Mora Vicente J, Ares Camerino A, Martínez González J. Desarrollo y aplicación de un cuestionario en una población escolar sobre el transporte de mochilas y su influencia en el dolor de espalda. Rev Pediatr Aten Primaria. 2004;6:199208.

19. Quintana Aparicio E, Martín Nogueras A, López Sendín N, Romero Alonso A, Sánchez Pe- draz L, Calvo Arenillas J. Influencia del tipo de jornada escolar en el peso de las mochilas escolares. Fisioterapia. 2005:27:6-15.

20. Román Viñas B, Serra Majem L, Ribas Barba L, Pérez Rodrigo C, Aranceta Bartrina J. Estimación del nivel de actividad física mediante el Test Corto Krece Plus. Resultados en la población española. En: Serra Majem L, Aranceta Bartrina J, Rodríguez-Santos $\mathrm{F}$ (eds.). Crecimiento y desarrollo. Estudio enKid, vol. 4. Masson: Barcelona, 2003.

21. Jones GT, Watson KD, Silman AJ, Symmons DP, Macfarlane GJ. Predictors of Low Back Pain in British Schoolchildren: A Population-Based prospective cohort study. Pediatrics. 2003;111:822-8.

22. Negrini S, Carabalona R, Sibilla P. Backpack as a daily load for schoolchildren. The Lancet. 1999;354:1974.

23. Giusti PH, De Almeida HL Jr, Tomasi E. Weight excess of school materials and its risks factors in South Brasil. A croos sectional study. Eur J Phy Rehabil Med. 2008;44:33-8.

24. van der Linden FJ, Dijkman TA. Jong zijn en volwassen worden in Nederland. Hoogveld Instituut Nijmegen. 1989:437:8. 